\title{
Impairments in the Comprehension of Indoor Object/Body Part Words and Family Relationships in a Patient with Aphasia due to Cerebral Infarction of the Left Parietal and Temporal Lobes
}

\author{
Nobuhiro Takahashi ${ }^{\mathrm{a}} \quad$ Minpei Kawamura $^{\mathrm{a}}$ Yasutaka Kobayashib $^{\mathrm{b}}$ \\ aDepartment of Rehabilitation, Fukui College of Health Sciences, Fukui, Japan; \\ ${ }^{b}$ Department of Rehabilitation Medicine, Fukui General Hospital, Fukui, Japan
}

\section{Keywords}

Aphasia $\cdot$ Cerebral infarction $\cdot$ Semantic category $\cdot$ Temporal lobe $\cdot$ Parietal lobe

\begin{abstract}
The present report discusses the case of a 73-year-old right-handed man who exhibited signs of aphasia caused by cerebral infarction in the left temporoparietal region. Neuropsychological evaluation revealed that he had difficulty in comprehending words related to indoor objects, body parts, and family relationships, compared to his vocabulary comprehension in other semantic categories. Although several reports have described cases in whom category-specific impairments in word comprehension are observed, few have documented cases in whom these impairments are accompanied by impairments in the comprehension of words indicating family relationships. Words in each of these three categories derive their meanings and notation
\end{abstract}




\section{Case Reports in Neurology}

Takahashi et al.: Semantic Category Impairments due to Aphasia

from spatial relationships. Therefore, it is likely that the patient's impairments were caused by difficulty in comprehending spatial relationships due to left parietal lobe damage.

(C) 2018 The Author(s)

Published by S. Karger AG, Basel

\section{Introduction}

Impairments in the comprehension of vocabulary items according to semantic category are reported to differ based on the location of brain damage. Idiosyncratic patterns of impairments in semantic category comprehension have been reported, including those in which patients had difficulty in comprehending the names of living organisms, man-made artifacts, tools, body parts, indoor objects, colors, and proper nouns. Yamadori et al. [1] presented a patient who exhibited impairments in comprehending the names of body parts and indoor objects, proposing the concept of word category aphasia. Since then, several reports [2-7] have discussed the comprehension of words for body parts and indoor objects, each suggesting that impairments in such comprehension are associated with left parietal lobe damage. Neuroimaging studies support the notion that different brain regions are involved in producing impairments associated with different semantic categories [8].

In this report, we discuss a patient who experienced difficulty in comprehending words for indoor objects and body parts, relative to other semantic categories. This patient also experienced difficulties in comprehending words describing family relationships. There are no reported cases of patients experiencing difficulties in comprehending words in these three semantic categories. Here, we examine the relationship between this patient's illness and such impairments in comprehension.

To assess impairments in the comprehension of body part words, we conducted a nonverbal body part orientation assessment to determine whether impairments occurred at the language level or in conjunction with nonverbal deficits in spatial comprehension. Impairments in the comprehension of words describing family relationships were assessed by examining relative changes in these terms.

\section{Case Presentation}

The patient was a 73-year-old right-handed man. One day prior to hospitalization, he began displaying abnormal behavior, such as crumpling the newspaper, attempting to put his cell phone into his cup of ramen, and uttering words such as "yes" and "why?" repeatedly. He was hospitalized the next day following a diagnosis of cardiogenic cerebral infarction. No motor, sensory, or cerebellar abnormalities were observed, although he exhibited signs of fluent aphasia, ideational apraxia, ideomotor apraxia, constructional apraxia, and optic ataxia. A diffusion-weighted MRI of the head taken on the second day of hospitalization showed hyperintensities in the left temporoparietal lobe (Fig. 1). The patient had no history of mental illness or neurological disorders. He had completed 16 years of education, and he attained his highest level of education at a 4-year university. He had retained his manners and seemed to understand words in the context of short sentences. His speech was fluent, and he could express his 


\section{Case Reports in Neurology}

Takahashi et al.: Semantic Category Impairments due to Aphasia

words in sentences, although he exhibited frequent paraphasia. Thus, his speech was difficult to understand because he did not correct himself.

\section{Neuropsychological Testing}

Standard Language Test of Aphasia

The Standard Language Test of Aphasia (SLTA) [9] (Table 1) is the most common comprehensive aphasia test in Japan. The test includes 26 subtests designed to assess each of the following language modalities: "listening," "speaking," "reading," "writing," and "calculating."

We conducted an initial assessment 2-3 days after hospitalization and a reevaluation 5865 days after hospitalization. SLTA results from the initial evaluation showed that word level language understanding was generally good. Language understanding at short sentence level was difficult. In the subtest of "speaking," word finding difficulty, phonemic paraphasia, and semantic paraphasia occurred frequently and speaking was extremely difficult. Spontaneous utterances were fluent, and he could speak in short sentences, although these utterances were empty due to omitted content words and frequent occurrences of paraphasia. In the writing domain, he scored $0 \%$ for describing a picture and dictation, indicating that he found this noticeably difficult.

In the reevaluation SLTA at 58-65 days following hospitalization, word level linguistic understanding remained good. For the short sentence level, the correct answer rate of auditory understanding improved to $80 \%$. However, the correct answer rate of his "follow verbal command" was $10 \%$. Five of the 10 short sentences presented in "follow verbal command" included spatial words such as "next to." He answered correctly when at least one hint was presented for short sentences without spatial words. In short sentences including spatial words, correct answers were not obtained with one hint. He continued to exhibit frequent instances of phonemic paraphasia and semantic paraphasia.

Test of Lexical Processing in Aphasia

The Test of Lexical Processing in Aphasia (TLPA) [10] (Table 2) assesses information processing at the word level in Japanese individuals. The TLPA includes a subtest of noun comprehension based on semantic category, divided into tests of listening comprehension and naming. The test includes 20 words associated with each of the following 10 semantic categories, for a total of 200 test items: indoor objects, buildings, vehicles, tools, processed foods, vegetables and fruit, plants, animals, body parts, and colors. In the auditory word recognition test, the patient is required to point to words that correspond to the auditory command from among 10 line drawings of the same category. For this auditory word recognition test, the $z$ score of each category was calculated based on the test data of 68 patients with aphasia.

We conducted an initial assessment 14 days after hospitalization and a reevaluation 63 days after hospitalization. The patient's correct answer rate 14 days after hospitalization was $35 \%$ for indoor objects $(z=-1.73)$ and $30 \%$ for body parts $(z=-1.87)$. The correct answer rate was particularly low for indoor objects and body parts.

The correct answer rate 63 days after hospitalization was $65 \%$ for indoor objects $(z=-0.30)$ and $60 \%$ for body parts $(z=-0.62)$. An overall improvement in the correct answer rate was observed, although he continued to have difficulty in understanding auditory words 


\section{Case Reports in Neurology}

Takahashi et al.: Semantic Category Impairments due to Aphasia

related to indoor objects and body parts. The correct answer rate of colors was 65\% $(z=-0.45)$, and the improvement was poor.

\section{Assessment of Nonverbal Body Part Orientation}

A decrease in the correct answer rate was observed in the body part category of the TLPA noun comprehension test. A nonverbal body part orientation assessment was performed to examine whether this language comprehension impairment represented an isolated language level issue, or whether it occurred in conjunction with an impairment in nonverbal spatial comprehension of body parts.

We conducted an initial assessment 15 days after hospitalization and a reevaluation 77 days after hospitalization. The first task required the patient to point to a part of his own body corresponding to the part to which the therapist pointed. Twenty body parts were chosen from among those in the TLPA noun comprehension test. Fifteen days after hospitalization, the patient scored 10/20 correct answers. However, he responded to all questions correctly 77 days after hospitalization.

During the second task, the patient was required to point to the portion of a picture corresponding to the body part indicated by the therapist (via pointing). Fifteen days after hospitalization, the patient scored 7/20 correct answers, although this improved to 19/20 correct answers 77 days after hospitalization.

Fifteen days after hospitalization, the patient was asked to draw a human body, but he only managed to draw a few lines. After 77 days, he was able to draw, albeit poorly, a discernible head, trunk, and upper and lower limbs (Fig. 2).

While bathing shortly after hospitalization, he would often fail to wash parts of his body that he could not directly see, such as his head and back, and required some assistance when taking baths. Around 70 days after hospitalization, he could wash body parts such as his head and back, and he was able to bathe independently.

Syntax Test of Aphasia

The Syntax Test of Aphasia (STA) [11] is used to assess Japanese syntax proficiency. The test consists of a listening comprehension test, a reading comprehension test, and a production test. The items of the listening comprehension and reading comprehension tests were constructed based on 5 levels of sentence comprehension: "level I: word meaning," "level II: word order," "level III: particle/complement clause (-)," "level IV: particle/complement clause $(+)$, " and "level V: relative clause/particle." Higher syntactic processing is required as the test progresses. "Level I: word meaning" is based on the meaning of content words, and it consists of questions at a level where the meaning of short sentences can be comprehended.

We conducted an initial assessment 29 days after hospitalization and a reevaluation 76 days after hospitalization. Twenty-nine days after hospitalization, the patient scored 5/8 correct answers for listening comprehension and reading comprehension for level I items. However, for sentences that included family relationships (e.g., "father was sitting"), all responses were incorrect $(0 / 3)$. Conversely, for sentences that did not include family relationships (e.g., "the boy is walking"), all responses were correct (5/5).

After 76 days of hospitalization, he answered all level I listening comprehension and reading comprehension questions correctly, and he was able to understand the sentences that included family relationships. 


\section{Case Reports in Neurology}

Takahashi et al.: Semantic Category Impairments due to Aphasia

Assessment of Family Relationship Comprehension

As the patient was suspected to have difficulty in comprehending family relationships based on his STA results, the following assessment was performed.

We conducted an initial assessment 30 days after hospitalization and a reevaluation 76 days after hospitalization. During the first task, the patient was required to select 1 person from among a 6-person family, in response to auditory questions regarding family relationships (e.g., "Who is the father?"). Thirty days after hospitalization, the patient scored 3/6 correct answers. He answered all questions correctly 76 days after hospitalization.

During the second task, the therapist presented a picture, following which the patient was required to point to the appropriate individual in a 6-person family, in response to questions such as "Who is this person's grandmother?" After 30 days of hospitalization, he scored 3/8 correct answers. After 76 days, he answered 5/8 correctly, indicating a slight improvement.

During the third task, the therapist presented the family tree of a nationally recognized anime protagonist (Fig. 3), following which the patient was required to point to the appropriate individual in response to questions such as "Who is this person's mother?" Thirty days after hospitalization, he scored 4/12 correct answers. Although improvement was observed after 76 days $(9 / 12$ correct), difficulties remained.

The second and third assessments differed from the first assessment in terms of the relative changes in names.

Wechsler Adult Intelligence Scale

To evaluate the nonverbal ability of our patient, we conducted the performance test of the Wechsler Adult Intelligence Scale (WAIS)-III [12]. An initial assessment was performed 5 days after hospitalization and a reevaluation 64 days after hospitalization.

Results on day 5 of hospitalization were performance IQ 63, perceptual organization 68, processing speed 52 , and a significant decrease in the nonverbal performance ability was recognized. Block design yielded an evaluation score of 1 (the average for healthy subjects of the same age is 10).

Results on day 64 of hospitalization were performance IQ 80, perceptual organization 85, and processing speed 69. Compared with the result of day 5 , the nonverbal performance ability had improved. The block design score improved to 6 .

\section{Discussion}

Our patient exhibited greater difficulty in listening comprehension when presented with words related to body parts and indoor objects, relative to words of other semantic categories. Such impairments are associated with damage to the left parietal lobe [3, 4]. Ito et al. [13] discussed a patient with semantic aphasia caused by Pick's disease, in which the parietal lobe is relatively spared from atrophy; atrophy progressed from the tip of the left temporal lobe, preserving the names of body parts until very late in the disease course. Such findings suggest that parietal lobe function and body part word comprehension and expression are linked. Yamadori [14] maintains that somatesthetic information from body parts is processed in the parietal lobe, and that such processing is associated with understanding the spatial relationships of each body part. In addition, Okudaira and Monoi [7] proposed that, for body part and 


\section{Case Reports in Neurology}

Case Rep Neurol 2018;10:181-192

DOI: $10.1159 / 000490938$

(c)

2018 The Author(s). Published by S. Karger AG, Base www.karger.com/crn

Takahashi et al.: Semantic Category Impairments due to Aphasia

indoor object words, the meanings of both types of words are formed based on part/part and part/whole relationships, further suggesting the involvement of the parietal lobe. Our patient had difficulty in grasping spatial relationships due to a cerebral infarction in the left temporoparietal lobe region, which likely caused the divergence in word recognition based on semantic category. As he also exhibited difficulties with nonverbal body part orientation in early assessments, we speculate that a collapse of the body schema had occurred as well. When reassessed at a later stage, he was almost able to locate body parts nonverbally and he no longer failed to wash specific body parts while bathing. Thus, impairment in body schema is considered to have improved. However, he continued to have difficulties in comprehending language associated with body parts, suggesting that semantic impairments related to the names of body parts continued. A decrease in the correct answer rate for auditory complementation of colors was also observed. We cannot explain the deterioration in the performance of color auditory complementation by the same mechanism as that for the understanding of body parts and indoor objects. It is therefore a limitation of this study.

Moreover, the patient also had difficulties in comprehending words indicating family relationships. Even during reassessment, he continued to have difficulty with relative changes in names (e.g., "[someone]'s grandmother"). Spatial images such as family trees are required to comprehend words that describe family relationships [15]. Suzuki [15] maintains that the functioning of visuospatial operations is mainly governed by the right parietal lobe, while language comprehension of spatial relationships is governed by the left parietal lobe. As this patient's illness included the left parietal lobe, we speculate that his difficulties in determining these spatial relationships resulted in difficulty in comprehending words associated with family relationships. More precisely, to evaluate the understanding of family relations, it was necessary to evaluate the ability to acquire the viewpoint of others and to infer verbal reasoning ability. These were further limitations of this case report.

We conducted the WAIS-III as an evaluation of nonverbal ability of our patient. A marked decline in nonverbal cognitive abilities in the WAIS-III initial assessment was noted. Although reevaluation of the WAIS-III was not enough, there was improvement in nonverbal cognitive ability. Based on the WAIS-III and the evaluation of body part localization, our patient had difficulty in grasping nonverbal spatial relationships at initial evaluation, but at reevaluation it was almost possible. From the reassessment of the SLTA's "Follow verbal command," we confirmed remarkable difficulty in understanding short sentences containing spatial words. Obstacles to understanding in short sentences including spatial words could support the finding of continued difficulty in speech understanding of spatial relations at reevaluation.

Our patient experienced impairments in the comprehension of words indicating indoor objects, body parts, and family relationships - all of which derive their meanings and notation from spatial relationships. Therefore, it is likely that difficulties in comprehending spatial relationships due to left parietal lobe damage resulted in difficulties in understanding words associated with these semantic categories.

\section{Conclusion}

The present report discussed the case of a patient with aphasia caused by cerebral infarction of the left temporoparietal region. The patient experienced difficulties in comprehending 
words indicating body parts, indoor objects, and family relationships, relative to words of other semantic categories. These impairments in language comprehension were thought to have stemmed from impairments in the comprehension of spatial relationships due to left parietal lobe damage.

\section{Statement of Ethics}

The authors have no ethical conflicts to disclose.

\section{Disclosure Statement}

The authors declare that they have no conflicts of interest to disclose.

\section{References}

1 Yamadori A, Albert ML. Word category aphasia. Cortex. 1973 Mar;9(1):112-25.

2 Dennis M. Dissociated naming and locating of body parts after left anterior temporal lobe resection: an experimental case study. Brain Lang. 1976 Apr;3(2):147-63.

3 Semenza C, Goodglass H. Localization of body parts in brain injured subjects. Neuropsychologia. 1985;23(2):161-75.

4 Goodglass H, Wingfield A, Hyde MR, Theurkauf JC. Category specific dissociations in naming and recognition by aphasic patients. Cortex. 1986 Mar;22(1):87-102.

5 Goodglass H, Budin C. Category and modality specific dissociations in word comprehension and concurrent phonological dyslexia. Neuropsychologia. 1988;26(1):67-78.

6 Fujimori M, Yamadori A, Imamura T, Yamashita H, Yoshida T. Category specific naming and comprehension impairment restricted to body parts and indoor house parts associated with a left parietal lesion. Jpn J Neuropsychol. 1993;9:240-7.

7 Okudaira N, Monoi H. Developing a Test of Lexical Processing in Aphasia (TLPA): an analysis of lexical deficits in aphasic patients. Higher Brain Function Res. 2000;20(3):234-43.

8 Damasio H, Grabowski TJ, Tranel D, Hichwa RD, Damasio AR. A neural basis for lexical retrieval. Nature. 1996 Apr;380(6574):499-505.

9 Japan Society for Higher Brain Dysfunction: Standard language test of aphasia. Tokyo: Shinko Igaku Syuppansya; 2006.

10 Fujita I, Monoi H, Okudaira N, Ueda M, Ono K, Shimogaki Y, et al. A test of lexical processing in aphasia. Tokyo: Esco Earl; 2000.

11 Fujita I, Miyake T. Syntax test of aphasia. Tokyo: Chiba Test Center; 1983.

12 Sugishita M. The Japanese version of the Wechsler Memory Scale-Revised. Tokyo: Nihon Bunka Kagakusha; 2007.

13 Ito K, Nakagawa Y, Ikeda M, Yamada N, Hashimoto M, Tanabe H. Category-specific word-meaning impairments in Gogi aphasics. Higher Brain Function Res. 1994;14(4):221-9.

14 Yamadori A. Categorical aspects in speech comprehension. Higher Brain Function Res. 1997;17(1):15-24.

15 Suzuki K. Neuropsychology of visual cognition. Tokyo: Igaku Shoin; 2010, p. 63-4. 


\section{Case Reports in Neurology}

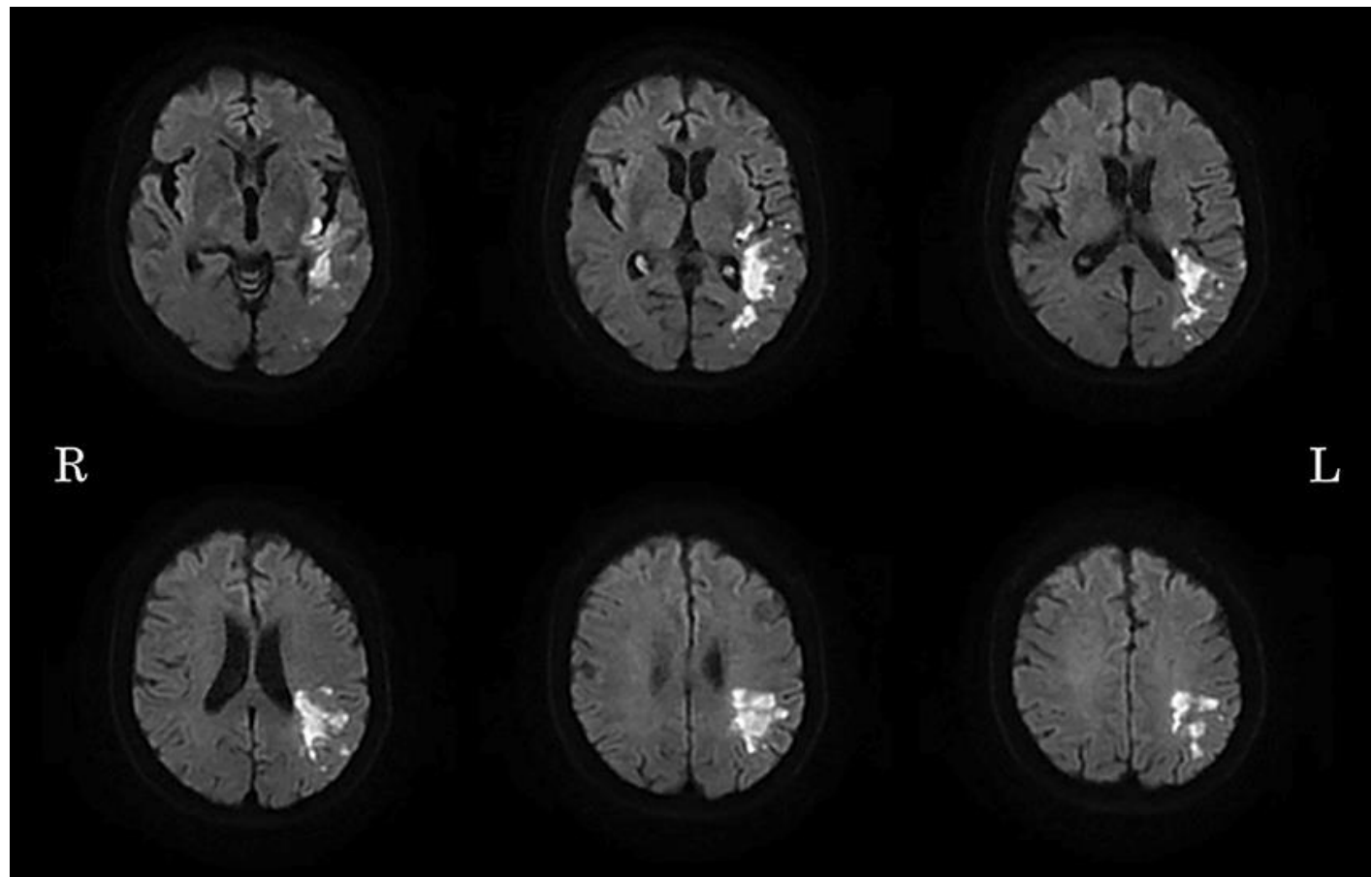

Fig. 1. Diffusion-weighted MRI of the head taken on the second day of hospitalization. Hyperintensities were observed from the left temporal lobe to the left parietal lobe. 


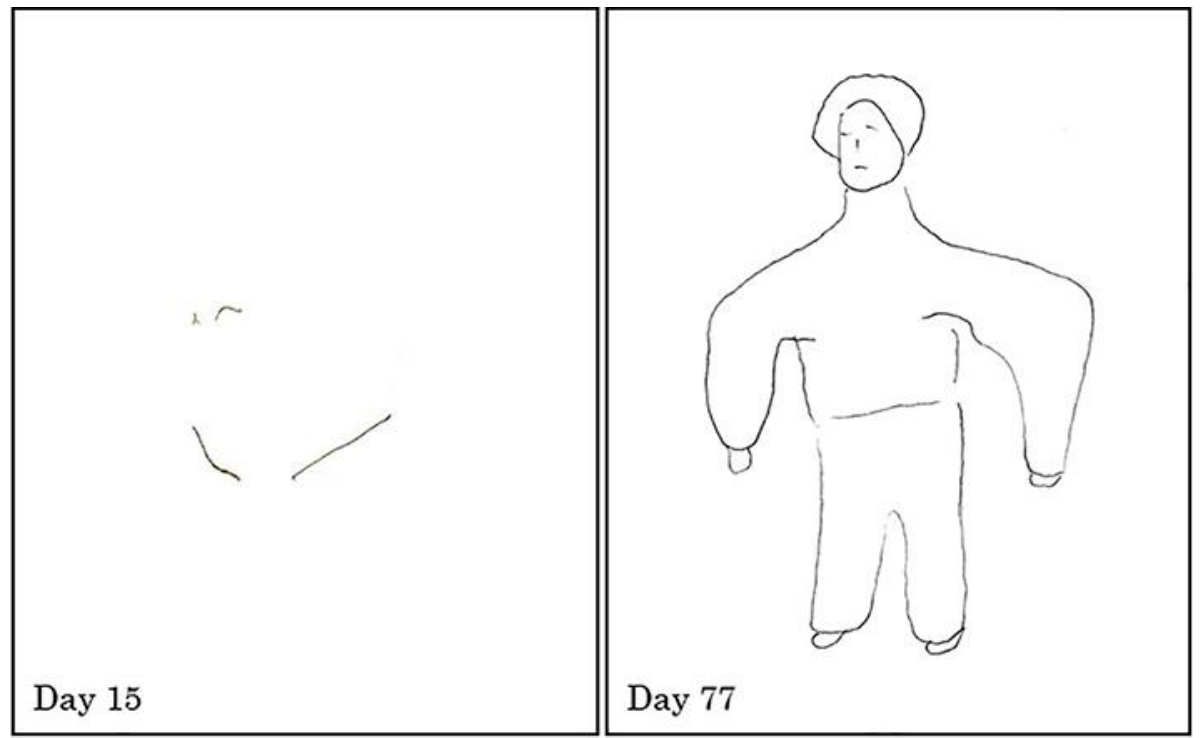

Fig. 2. Drawing of a human body. The patient's drawing 15 days after hospitalization (left image) consisted of only a few lines. Drawing was not possible for him. After 77 days (right image), he was able to draw, albeit poorly, a discernible head, trunk, and upper and lower limbs. 


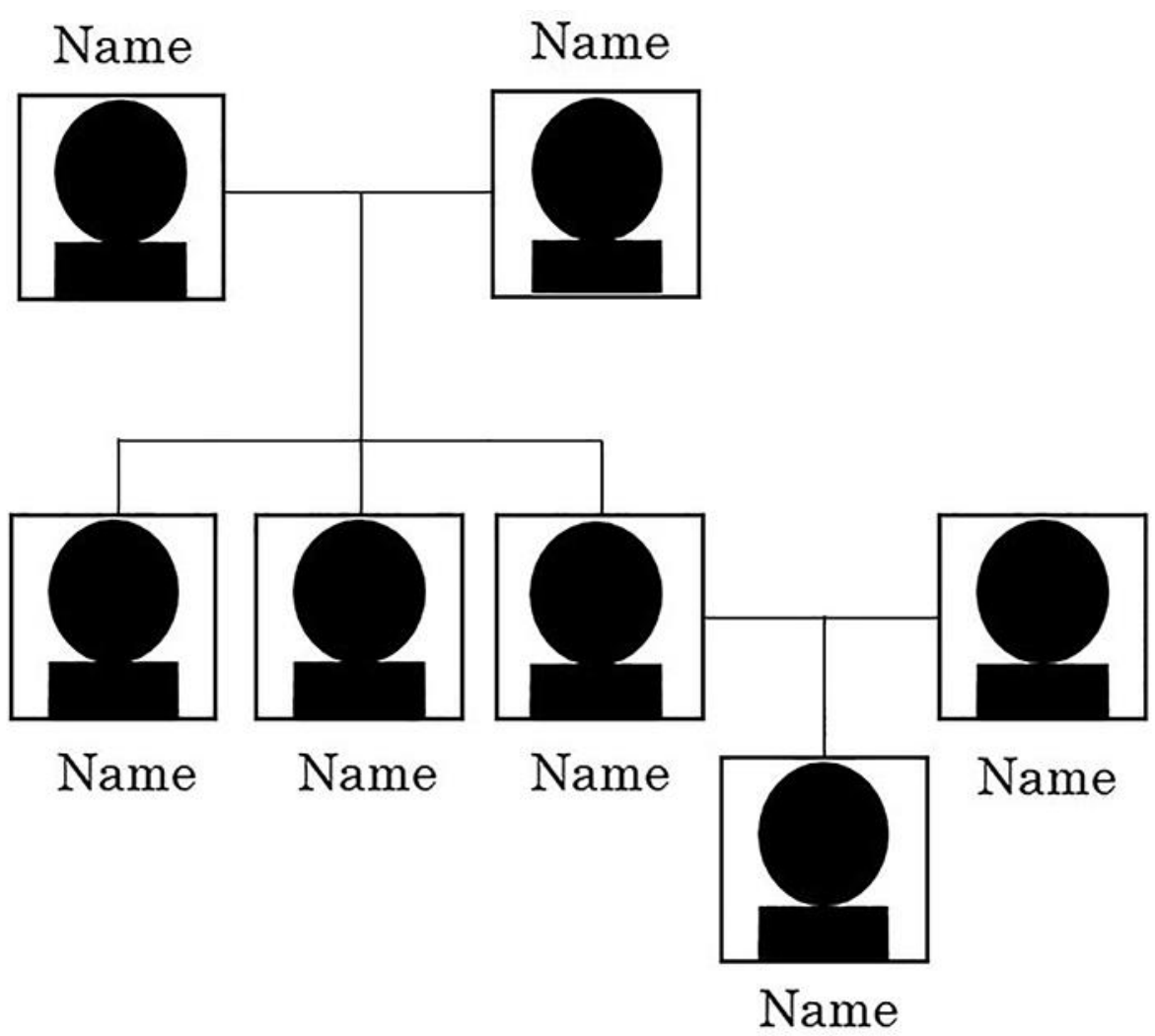

Fig. 3. Family tree presented for the assessment of family relationship comprehension. The names and faces of the characters were presented at the time of actual assessment. 
Table 1. Standard Language Test of Aphasia

\begin{tabular}{|c|c|c|c|c|}
\hline & & & $\begin{array}{l}\text { Percentag } \\
\text { answers }\end{array}$ & of correct \\
\hline & & & days $2-3$ & days $58-65$ \\
\hline I & 1 & Auditory word recognition & 100 & 100 \\
\hline & 2 & Sentence comprehension & 50 & 80 \\
\hline & 3 & Follow verbal commands & 0 & 10 \\
\hline & 4 & Kana letter discrimination & 30 & 80 \\
\hline II & 5 & Speaking object naming & 0 & 40 \\
\hline & 6 & Word repetition & 20 & 50 \\
\hline & 7 & Describe behaviors & 20 & 30 \\
\hline & 8 & Explain picture story & 0 & 20 \\
\hline & 9 & Sentence repetition & 0 & 0 \\
\hline & 10 & Word fluency & 0 & 20 \\
\hline & 11 & Read aloud kanji words & 40 & 60 \\
\hline & 12 & Read aloud kana letters & 0 & 60 \\
\hline & 13 & Read aloud kana words & 60 & 100 \\
\hline & 14 & Read aloud short sentence & 0 & 0 \\
\hline III & 15 & Kanji word-picture matching & 100 & 100 \\
\hline & 16 & Kana word-picture matching & 90 & 100 \\
\hline & 17 & Sentence-picuture matching & 60 & 60 \\
\hline & 18 & Follow written commands & 0 & 0 \\
\hline IV & 19 & Write kanji words & 0 & 20 \\
\hline & 20 & Write kana words & 0 & 0 \\
\hline & 21 & Narrative writing & 0 & 0 \\
\hline & 22 & Dectate kana letters & 0 & 0 \\
\hline & 23 & Dectate kanji words & 0 & 0 \\
\hline & 24 & Dectate kana words & 0 & 0 \\
\hline & 25 & Dectate short sentence & 0 & 0 \\
\hline V & 26 & Calculation & 0 & 5 \\
\hline
\end{tabular}

I: listening; II : speaking; III : reading; IV: writing; V: calculating. 
Table 2. Test of Lexical Processing in Aphasia (TLPA): noun recognition based on semantic category

\begin{tabular}{|c|c|c|c|c|c|c|c|}
\hline & \multirow{2}{*}{$\begin{array}{l}\text { All } \\
\text { words, } \\
n\end{array}$} & \multicolumn{3}{|l|}{ Day 14} & \multicolumn{3}{|l|}{ Day 63} \\
\hline & & $\begin{array}{l}\text { correct } \\
\text { answers, } \\
n\end{array}$ & $\begin{array}{l}\text { correct } \\
\text { answers, } \\
\%\end{array}$ & $\begin{array}{l}Z \\
\text { score }\end{array}$ & $\begin{array}{l}\text { correct } \\
\text { answers, } \\
n\end{array}$ & $\begin{array}{l}\text { correct } \\
\text { answers, } \\
\%\end{array}$ & $\begin{array}{l}Z \\
\text { score }\end{array}$ \\
\hline Indoor objects & 20 & 7 & 35.0 & -1.73 & 13 & 65.0 & -0.30 \\
\hline Structures & 20 & 15 & 75.0 & -0.39 & 20 & 100.0 & 0.93 \\
\hline Vehicles & 20 & 19 & 95.0 & 0.45 & 20 & 100.0 & 0.80 \\
\hline Tools & 20 & 16 & 80.0 & -0.35 & 17 & 85.0 & -0.09 \\
\hline Processed foods & 20 & 17 & 85.0 & -0.15 & 19 & 95.0 & 0.47 \\
\hline Vegetables and fruits & 20 & 16 & 80.0 & -0.25 & 18 & 90.0 & 0.29 \\
\hline Plants & 20 & 14 & 70.0 & -0.45 & 19 & 95.0 & 0.68 \\
\hline Animals & 20 & 20 & 100.0 & 0.64 & 20 & 100.0 & 0.64 \\
\hline Body parts & 20 & 6 & 30.0 & -1.87 & 12 & 60.0 & -0.62 \\
\hline Colors & 20 & 11 & 55.0 & -0.90 & 13 & 65.0 & -0.45 \\
\hline Total amount & 200 & 141 & 70.5 & -0.67 & 171 & 85.5 & 0.20 \\
\hline
\end{tabular}

$z$ scores were calculated based on the test data of 68 patients with aphasia, as indicated in the TLPA manual. 